\title{
57. Studien über die Chemischen Veränderungen des Blutes bei experimentellem Sonnenstich.
}

\author{
Von Rokuro SEkiguchi. \\ Medizinische Klinik von Prof. Inada, Kaiserliche Universität, Tokyo.
}

(Comm. by R. INADA, M.I.A., April 12, 1930.)

Von Juli bis Oktober 1929 beschäftigte ich mich mit den Studien über den Sonnenstich, wobei hauptsächlich die Veränderung des Milchsäuregehalts, nebenbei das Verhalten von Wasserstoffionenkonzentration und Alkalireserve, sowie den Zucker- und Chlorgehalt des Blutes berücksichtigt wurden.

Zum Versuch dienten etwa $2 \frac{1}{2}$ Kilo schwere Kaninchen mit grossen Ohren. Sie wurden in einen mit einem gewissen Apparat versehenen Kasten hineingebracht und der Sonne ausgesetzt, bis Krankheitssymptome erschienen. Dann wurden sie aus dem Kasten herausgenommen, (Körpertemperatur dabei in den meisten Fällen über $44^{\circ} \mathrm{C}$ ) Blut entnommen und die oben genannten Substanzen quantitativ bestimmt. Als Kontroll wurde das Blut derselben Tiere vor den Experimenten untersucht und zum Vergleich benutzt.

Zur Bestimmung von Wasserstoffionenkonzentration und des $\mathrm{CO}_{2-}$ bindungsvermögens kam das Plasma des unter flüssigem Paraffin entnommenen und zentrifugierten Blutes zur Verwendung. Da wurde die Wasserstoffionenkonzentration nach Cullen, das $\mathrm{CO}_{2}$-bindungsvermögen mit dem van Slykeschen Apparat, die Milchsäure nach der Mikrokolorimetrie von Mendel und Goldscheider gleich nach der Blutentnahme, der Blutzucker nach Hagedorn und Jensen, das Chlor nach Rusznyak bestimmt.

1. Bei Kaninchen mit experimentellen Sonnenstich findet man eine auffallende Vermehrung der Blutmilchsäure in sämtlichen Versuchsfällen (15 Fälle) ohne Ausnahme; der Minimalwert betrug $70 \mathrm{mg} \%$, der Maximalwert $180 \mathrm{mg} \%$, der Durchschnittswert $122,6 \mathrm{mg} \%$. Die Vergleichung der Körpertemperatur mit der Milchsäuremenge hat ergeben, dass bei der Erhöhung der ersteren die letztere gesteigert war. Bei den Kontrolltieren vor dem Experiment betrug die Blutmilchsäure in minimo $17,5 \mathrm{mg} \%$, in maximo $59,5 \mathrm{mg} \%$, im Durchschnitt (von 15 Fällen) $35,7 \mathrm{mg} \%$. Solche dreifache Milchsäurezunahme in den Durchschnittswerten lässt sich durch einen Einfluss 
der zur Zeit der Versuche unvermeidlichen Muskelbewegung der Kaninchen nicht erklären, sie ist auf nichts anderes zurückzuführen, als auf eine abnorme Steigerung des intermedialen Stoffwechsels des Kohlenhydrates im Körper des Kaninchens durch hohe Temperatur.

2. Auch das $\mathrm{Ph}$ des Blutplasmas geht bei experimentellem Sonnenstich bedeutend nach der sauren Seite über. Es betrug beim Kontrollversuche in minimo $\mathrm{Ph} 7.16$, in maximo $\mathrm{Ph} 7.36$, im Durchschnitt (von 15 Fällen) $\mathrm{Ph} 7.26$; nach der Erkrankung am Sonnenstich in minimo $\mathrm{Ph}$ 6.91, in maximo $\mathrm{Ph} 7.26$, im Mittel $\mathrm{Ph}$ 7.14; die Zunahme der Acidität im Durchschnitt 0.12.

3. Das $\mathrm{CO}_{2}$-bindungsvermögen des Blutplasmas des Kaninchens betrug in meinen Fällen beim Kontrollversuche in minimo 42.1 vol. \%, in maximo 69.0 vol. \%, im Durchschnitt (von 15 Fällen) 52.5 vol. \%; nach der Erkrankung am Sonnenstich zeigte es je nach der Schwere der Symptome verschiedene bedeutende Verminderung: in minimo 9.0 vol. \%, in maximo 56.0 vol. \%, im Durchschnitt 31.8 vol. \%, d. h. im Durchschnitt eine Abnahme um 20.7 vol. \%, welche einen ausgesprochenen Mangel an Alkalireserve im Kaninchenblut verursacht, der seinerseits zu einer Störung des Säurebasengleichgewichts im Organimus führt. Diese Verminderung des $\mathrm{CO}_{2}$-bindungsvermögens scheint meist in geradem Verhältnis zu der Vermehrung der Milchsäure und der Erhöhung der Wasserstoffionenkonzentration zu stehen, aber hier sind keine distinkten numerischen Verhältnisse zu ermitteln.

4. Blutzuckerveränderung. Im Allgemeinen erfährt die Menge des Blutzuckers zur Fieberzeit eine Vermehrung. Nach Imazu werden die Blutzuckerwerte bei der Erkrankung am Sonnenstich grösser. Auch in meinen Fällen waren sie beim Kontrollversuche in minimo 0.073 , in maximo 0.136, im Mittel (von 15 Fällen) 0.106 ; nach der Erkrankung zeigten 13 von 15 Fällen bedeutende Hyperglykämie, während 2 (Fall 11 u. 13) im Gegensatz Hypoglykämie erkennen liessen, welche als eine Erscheinung der Agonie zu betrachten ist. In den meisten Fällen fand sich Hyperglykämie; die Minimalwerte betrugen $0.127 \%$, die Maximalwerte $0.231 \%$, die Durchschnittswerte $0.145 \%$.

5. Über die Menge des Blutplasma-NaCl.

Bei meinen Versuchen wurde die Chlormenge nur in 5 Fällen (Fall 11-15) bestimmt. Eine so geringe Zahl von Bestimmungen gestattet es nicht, daraus Schluss zu ziehen, aber sie war bald vermehrt, bald fast unverändert, bald vielmehr vermindert, also sehr 
schwankend und variabel. Bei 5 normalen Kontrolltieren betrug sie im Mittel $0.556 \mathrm{~g} / \mathrm{dl}$, nach der Erkrankung $0.550 \mathrm{~g} / \mathrm{dl}$.

Kaninchen, bei denen der Blutmilchsäuregehalt nach der Erkrankung niedrig war, zeigten meistens einen milderen Verlauf, so dass sie nicht sofort starben. Aber sie gingen alle einige Tage später endlich zugrunde.

An einigen (Fall 5, 8, 12, 14) schwerverlaufenden Fällen wurde nach 1 oder 2 Stunden nochmals eine Blutuntersuchung vorgenommen, welche ergab, dass die Milchsäure in 4 Fällen eine noch stärkere Vermehrung erfuhr und dass auch die anderen Veränderungen parallel stärker auftraten.

Der experimentelle Sonnenstich ruft eine Verändrung am verschiedenen Stoffwechsel d. h. die Produktion saurer Substanz bes. der Milchsäure, des intermediären Kohlehydratstoffwechsels und damit eine Verminderung des Alkalireserves im Blut hervor, welche Veränderungen klinisch sog. Acidosis vorstellen, die mit schweren Symptomen wie Hyperpnoe, Krampfanfall usw. einhergeht.

Es ist noch nicht $\mathrm{zu}$ entscheiden, ob diese Acidosis in allen Fällen als Milchsäureacidosis bezeichnet werden kann, aber es unterliegt keinem Zweifel, dass die Milchsäure ihr wesentlicher Faktor ist.

Hier gehen die Veränderungen des Blutes fast ausnahmslos alle nach einer bestimmten Richtung $d$. h., auf die acidotische Seite über, und je stärker die Veränderungen sind, desto schwerer sind die klinischen Symptome, wie es scheint.

Zwischen der Vermehrung der Milchsäure, der Zunahme der Wasserstoffionenkonzentration und der Verminderung des Alkali-reserves sind keine deutlichen numerischen Verhältnisse zu erkennen, aber diese drei Veränderungen scheinen fast parallel $\mathrm{zu}$ gehen. 